\title{
Performance Evaluation Of Fuzzy Logic ANd Back Propagation Neural Network For Hand WRITTEN CHARACTER RECOGNITION
}

\author{
Heba M. Abduallah and Safaa S. Mahdi \\ Department of Computer Engineering, Al- Nahrain University, Baghdad, Iraq
}

\begin{abstract}
Fuzzy c-mean is one of the efficient tools used in character recognition. Back propagation neural network is another powerful that may be used in such field.

A comparison between fuzzy c-mean and BP neural network classifiers are presented in this research to obtain the performance of both classifiers. The comparison was based on recognition efficiency; this efficiency was evaluated as the ratio of the number of assigned characters with unknown one to the number of character set related to that character. The fuzzy C-mean and BP neural network algorithms were tested on a set of hand written and machine printed dataset named Chars $74 K$ dataset using Matlab (2016 b) programming language and the result was that neural network classifier gave $82 \%$ recognition efficiency while fuzzy c-mean gave 78\%. Neural network classifier is more superior than fuzzy C-mean in recognition due to the limitations of processing time of fuzzy $C$-mean that requires smaller image size and eventually this will cause less efficiency.
\end{abstract}

\section{KEYWORDS}

Fuzzy c-mean, character recognition, Back propagation neural network, recognition efficiency \& Chars $74 K$ dataset

\section{INTRODUCTION}

During previous years, many studies and applications was utilized for handwritten recognition of text in documents and PDF images [1]. Character recognition is a process of identifying printed symbol individually. These symbols can be alphabetic, numeric or punctuation etc. They also may be written either by hand or by machine (i.e. computer, tab, phone...etc.) in different fonts and sizes. More accurately character recognition is the process of recognizing and detecting input image characters then converting them into machine editable form [2]. Machine printed characters are more uniform and separated than handwritten characters which are non-uniform and their size and shape depending on the pen pressure by the writing person, even handwriting of same person may be changed depending on the situation in which the person writes. Many writing styles results in the distortion of characters from the standard characters used for the training of the system, which gives false results. That is the reason of the difficult task for designing a system that is capable of identifying the character with $100 \%$ accuracy.

In this paper a comparison between Fuzzy C-Mean and Back Propagation Neural network classifiers on the $\mathrm{Ch} 74 \mathrm{~K}$ dataset [3] are presented to obtain their performance in recognition. In this dataset, characters, digits, and symbols are available in the English language, Latin script (without accents) and Hindu-Arabic numerals. Simply it is called as the "English" characters set. This dataset contains:

- 62 classes (0-9, a-z, A-Z).

DOI : $10.5121 /$ ijesa.2018.8402 
- 7705 natural images' characters.

- 3410 handwritten characters written by using tablet PC.

- 62992 computer fonts' characters.

This gives a total of over 74000 images (that is why this dataset is called $74 \mathrm{k}$ ), but only English characters and digits were used which give us 62 classes.

To limit the time required for classification, preprocessing was done for the dataset images by converting the colored images into gray scale, and then features were extracted from them using wavelet transform which is used in many applications [4] as in remote sensing, texture analysis, data compression, image processing, medical image technology, and pattern recognition. Wavelets allows complex information such as patterns, speech, and images to be decomposed into their primary forms at different scales and positions, and then reconstructed with great precision. Only approximation coefficient features was used for the next steps.

Wavelet image features were size normalized prior to classification to have better recognition efficiency, then classification was done by Fuzzy and Neural classifiers, Fuzzy C-Means (FCM) [5], is a simple and statistical feature comparison of pixel features that characterize the object distinctively. Fuzzy Clustering uses an unsupervised learning approach, by identifying the number of clusters in the data; clustering is done by exploring the data structure then partitioning it into clusters automatically. This procedure typically results in a set of centroids which represents the types of data clusters, and they are used for further classification. Clustering techniques make a 'hard' partition on the data which is suitable for separated data like English characters.

Neural Networks [6] advantages are their adaptive-learning, self-organizing and fault-tolerance abilities. For these abilities, they are used for applications of pattern recognition. Neural Network, builds a non-limited distinguished surface border, for its input. This surface is constructed gradually by searching through the identified information from labeled patterns in the process of training. The trained network is then used later to classify other patterns by using the learned information during training. The distinguished surface is converted into values of activation function threshold and weights of the networks while training.

Finally performance evaluation of two classifiers based on recognition efficiency was done, this efficiency which was evaluated as the ratio of the number of assigned dataset training characters with test characters to the number of character set related to that one.

Many researches have been done in the previous years on character recognition system including, different methods of preprocessing, segmentation, feature extraction and feature classification. These methods recently headed towards artificial neural network, genetic algorithms and deep learning [7], [8], [9].

In 2014, Siddhi Sharma et al. [10], used a single-level two-dimensional wavelet decomposition wavelet called Symlet 'sym4'for feature extraction. This wavelet calculates the approximation and detailed coefficients of the image. They estimated six geometrical features like standard deviation, mean, median, minimum, maximum, and variance for these coefficients. Later a Back Propagation Neural network classification wass applied using Matlab toolbox on 25 samples for each character, and a recognition efficiency of $84.8 \%$ was resulted for testing ten characters from these samples.

In 2014, S.V. Shelke et al. [11], collected 13 sets of handwritten characters written by different users; feature extraction was done by using two dimensional multilevel wavelet transform method. Wavelet families used were Daubechies and Reverse Bi-orthogonal. Three levels up to 6, 7, 8th wavelet decomposition was done. The obtained Features were then trained in WEKA3.6 machine 
learning software using different classifiers like Multilayer perceptron, K-Nearest Neighbor. Results for different classifiers were calculated that multilayer perceptron gave $92 \%$ accuracy for 8th level decomposition, $98 \%$ for 6th level decomposition.

\section{FuZZY C-MEANS}

A Fuzzy classifier is based on Fuzzy C-Means [5] method. Let $A$ be a vector's set $x_{k}, k=$ $1,2, \ldots . n, n$ is image blocks' number that membership function of them to be determined,

$$
A=\left\{x_{1}, x_{2}, \ldots . x_{n}\right\}
$$

Grouping of pixel blocks depending to their membership and regions results in a membership function,

$$
U=\left[u_{i k}\right], 1 \leq i \leq c, 1 \leq k \leq n
$$

Where $U$ is the membership matrix for the pixel block $k$ in $i$ region, and $c$ is the number of regions.

The elements of $U$ should satisfy the following conditions:

$$
\begin{gathered}
\sum_{i=1}^{c} u_{i k}=1, \quad \forall k ; \\
\sum_{i=1}^{c} u_{i k}>0, \quad \forall i ; \\
u_{i k} \in[0,1], \quad \forall i, k
\end{gathered}
$$

$u_{i k}$ Value is the probability that a block $k$ is mapped into a region $i$. The Fuzzy C-Means method reduces the waste of feature properties between region $i$ center and $k$ block for the determination of the pixel membership as shown below:

$$
J_{m}(U, V)=\sum_{k=1}^{n} \sum_{i=1}^{c} u_{i k}^{m} d_{i k}^{2}=\sum_{k=1}^{n} \sum_{i=1}^{c} u_{i k}^{m}\left\|x_{k}-v_{i}\right\|^{2}
$$

Where $m \gg 1$, used to control the fuzziness of the cluster, i.e. $m=1$ means that all regions possess high fuzziness; matrix $V=\left[v_{1}, v_{2}, \ldots, v_{c}\right]$ holds the vectors where it represents the center of each region, and $d_{i k}$ denotes the dispersion of $x_{k}$ from $v_{i}$, i.e. $d_{i k}=\left\|x_{k}-v_{i}\right\|$ and $d_{j k}=\| x_{k}-$ $v_{j} \|$.

Minimizing the function $J_{m}$ is done by updating the values of $u_{i k}$ and $v_{i}$ repeatedly according to the relation below:

$$
\begin{aligned}
u_{i k} & =\left(\sum_{j=1}^{c}\left(\frac{d_{i k}}{d_{j k}}\right)^{\frac{2}{m-1}}\right)^{-1}, \forall i, k \\
V_{i} & =\frac{\sum_{k=1}^{n} u_{i k}^{m} x_{k}}{\sum_{k=1}^{n} u_{i k}^{m}}, \forall i
\end{aligned}
$$

$v_{i}$ and $d_{i k}$ value is obtained from the above relative equations, and by setting the iteration tolerance to a certain value, while initial error is small, the membership of $x_{k}$ in region $i$ is repeatedly adjusted by a new value of $U$, which called $U^{\text {new }}$ as shown below:

$$
\text { error }=\left\|U^{\text {new }}-U^{\text {old }}\right\|=\max \left\|u_{i k}^{(\text {new })}-u_{i k}^{(\text {old })}\right\|, 1 \leq i \leq c, 1 \leq k \leq n
$$


As $J_{m}$ is iteratively minimized, $v_{i}$ became more stable. Iteration stops when the error is smaller than the iteration tolerance.

The algorithm of Fuzzy C-mean can be summarized as follows:

2.1 Pixel by pixel fuzzification was done, by

- The input is normalized (for fuzzy normalization to $8 * 8$ ) gray scale unknown characters and normalized gray scale dataset characters.

- Set maximum iteration to $5, m=2$ and maximum error $=5^{*} 10^{-3}$.

- Initializing the center, $V_{i}$ by choosing unknown character/digit to be the center. The size of each dataset images and unknown character was set to $8 * 8$ (64).

- Calculating the membership function, $u_{i k}$ using equation (7).

- Calculating the new center, $V_{i}$ by using equation (8).

- Updating the membership function value with the new center by using equation (7).

- Stopping criteria, by calculating the error value using equation (9). If the calculated error < maximum error, then it stops and moving to the next pixel, else a second iteration is begun and all steps in step (2) is repeated.

- If the iteration number exceeds 5, the algorithm will assume that unknown feature isn't related to the known feature.

2.2 The recognition efficiency is calculated by calculating the number of correctly identified characters/digits, using the (number of matched features/64) which give us the recognition efficiency of each character with the whole database.

\section{Back Propagation Neural Network}

Back Propagation Neural Network (BPNN) [10] algorithm is the most used algorithm. For the BPNN input and target features are used to train a network until it approximates a function, aid of the input features with specific output features, or classifying of input features in a suitable way as needed.

Back Propagation Algorithm steps are,

\subsection{Training AlgorithM:}

\subsubsection{THE INITIALIZATION OF WEIGHTS:}

All weights were set into a few of small randomly selected values.

\subsubsection{The CAlCUlation OF ACtivation FunCtion:}

Log-sigmoid activation function denoted as logsig activation function [12], $f$ which was calculated by the following equation,

$$
f(n e t)=\frac{1}{1+e^{-n e t}}
$$

\subsubsection{AT THE INPUT:}

Determination of the Activation function of the input was by the samples offered to the network. 


\subsubsection{AT HIDDEN AND OUTPUT:}

Determination of the Activation function $O_{j}$ of Hidden and Output by following equation,

$$
O_{j}=f\left[\sum w_{i j} * O_{i}-\theta_{j}\right]
$$

Where:

$w_{i j}$ : input $O_{i}$ to unit $j$ weights.

$\theta_{j}$ : unit $j$ node threshold

$f$ : The Activation Function

3.1.3 Training of the Weights

\subsubsection{Changing Weights:}

In this step, it starts at output then working in reverse to the hidden layer, and weight adjusting by following equation,

$$
w_{j i}(t+1)=w_{j i}(t)+\Delta w_{j i}
$$

\subsubsection{COMPUTATION OF WEIGHT ChANGE:}

Computing the weight change by following equation,

Where $\mathrm{n}$ : is the learning rate, $\Delta w_{j i}=\eta \delta_{\mathrm{j}} \mathrm{O}_{\mathrm{j}}$

$\delta_{\mathrm{j}}$ : is the error gradient

The error gradient can be computed at the Output Unit as follows,

$$
\delta_{j}=O_{j}\left(1-O_{j}\right)\left(T_{j}-O_{j}\right)
$$

And for the Hidden Unit

$$
\delta_{j}=O_{j}\left(1-O_{j}\right) \sum \delta_{k} w_{k j}
$$

Where

$T_{j}:$ is the Target Value,

$O_{j}$ : is the Actual Output Value,

$\delta_{k}:$ is the Error Gradient $\mathrm{k}$ at connection point $j$.

3.1.5 Repeating the iterations until convergence.

The algorithm used $\mathrm{Ch} 74 \mathrm{~K}$ dataset characters that were divided into a $70 \%$ train set and $30 \%$ test set, which gives a total of (51928 training images, 22177 test images). Then the test images was divided so that each character samples individually isolated with labels for each one, in addition to labels for the training set.

- Neural network consists of training then testing, the training algorithm can be summarized in the following steps:

- The input is the normalized $(32 * 32)$ gray scale training images and their labels, input layer size which is $(32 * 32=1024)$, hidden layer size $=200$, number of labels $=$ 62 , random weight initialization, maximum iteration $=1000$, $\max$ error $=5^{*} 10^{-3}$, and learning rate $=0.7$. 
- The updated weights were calculated using equation (12).

- The error was calculated using equation (14).

- When the updated weights reached the max error allowed, next iteration begun until maximum iteration is reached.

- The updated weights after training were saved to be used in testing algorithm.

- The testing algorithm used can be summarized in the following steps:

$\circ$ The input is the normalized $(32 * 32)$ gray scale test images and their labels, input layer size $=1024$, hidden layer size $=200$, number of labels $=62$, and the updated weights from the training algorithm.

$\circ$ The activation function was calculated using equations (10 and 11).

- A prediction for the test characters was calculated by comparing the test labels for the test images, with the train labels for the output of the activation function.

- The recognition efficiency was estimated by calculating the number of correctly identified labels to the total number of dataset labels.

\section{RESULTS}

In this paper, two methods for character classification were presented, and then a comparison of their recognition efficiency on Char74K dataset was done. These two methods were Fuzzy CMeans and Back Propagation Neural Network.

4.1 Initially, the dataset images including train and test images is preprocessed to have a gray level images see figure (1), to have less features than when using colored image so as to reduce the time consumed in classification and to have a better efficiency than using binary images.

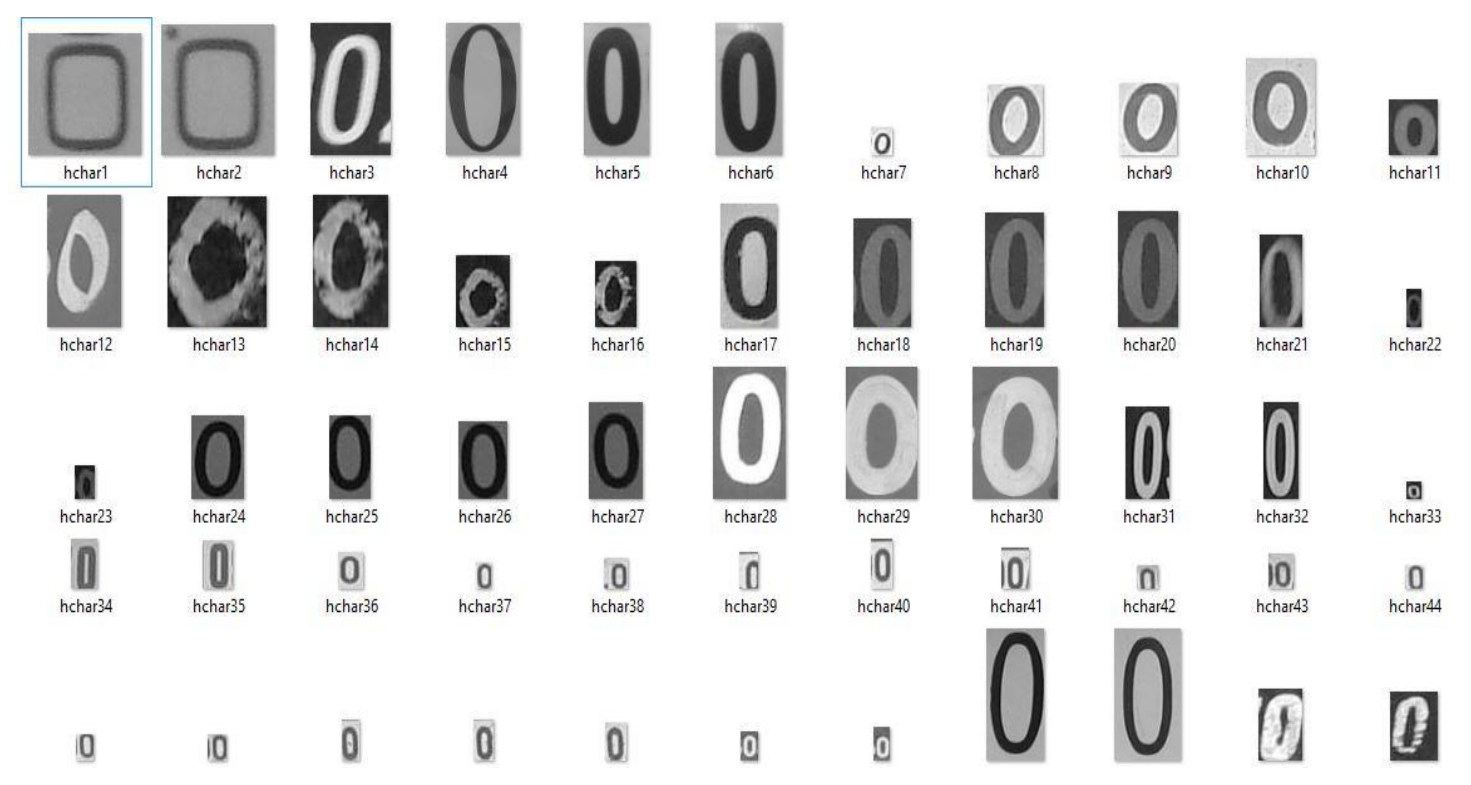

Figure 1. The conversion of images into gray level

4.2 The feature extraction is performed using frequency domain wavelet transform as shown in figure (2), frequency features is resulted and only the approximation coefficients (LL) features is used for classification to reduce the number of features to be classified so as to reduce classification time as possible. 


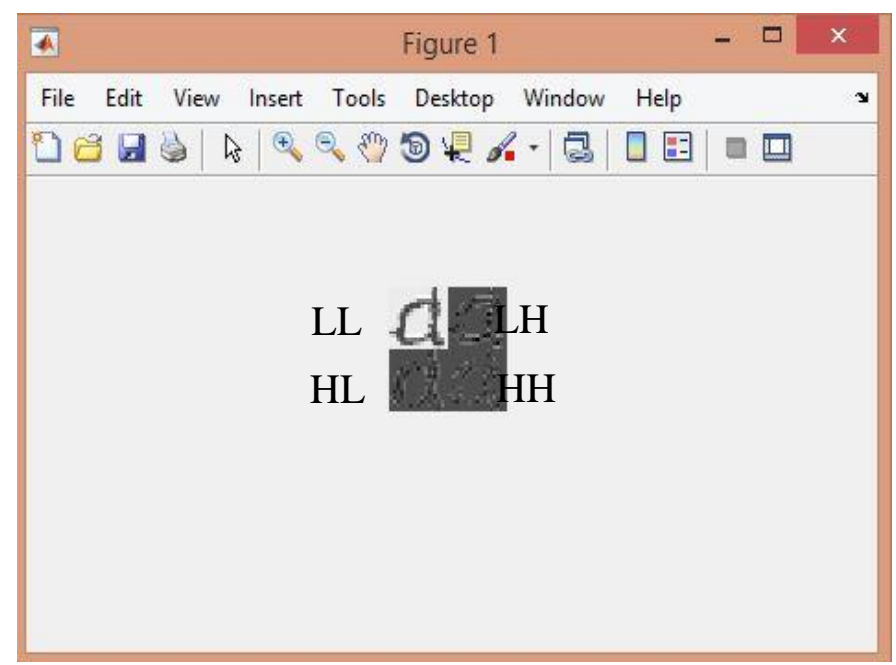

Figure 2. Wavelet transforms for character (a) from the dataset images

4.3 These approximation features were resized into ( $8 * 8$ pixel), for Fuzzy classification as fuzzy pixel by pixel classification requires more time to perform fuzzification process (i.e. fuzzification means Fuzzy algorithm process mentioned in section (II) of this paper) as the time consumed for one character was less than using $\left(16^{*} 16\right.$ pixel) because number of pixels was $\left[16^{*} 16^{*} 5\right.$ (no. of iterations $)=1280$ pixel], while for $8 * 8$ it was $(8 * 8 * 5=320$ pixel $)$ and when using $(4 * 4)$ less time consumed for recognition $(4 * 4 * 5=80$ pixel) but the character image cannot be recognized even by human eye because of its low resolution, which leads to lower recognition efficiency.

4.4 While size normalization was $(32 * 32$ pixel) for Neural classification, as when using $(16 * 16$ pixel) the recognition efficiency was reduced by nearly $10 \%$, and when using $(64 * 64$ pixel) the training time consumes more time and the training process is not terminated yet. While neural classification using $(32 * 32)$ as it requires only time for the training of the network and the network only trained once and doesn't consume much time for test.

4.5 The error limit was $5^{*} 10^{-3}$ depending on changing the error values until having the best possible efficiency.

4.6 Then recognition efficiency is calculated for the Fuzzy and Neural classification and a comparison is made between them. Figure (3) shows recognition efficiency comparison for some characters using Fuzzy and Neural classification. 


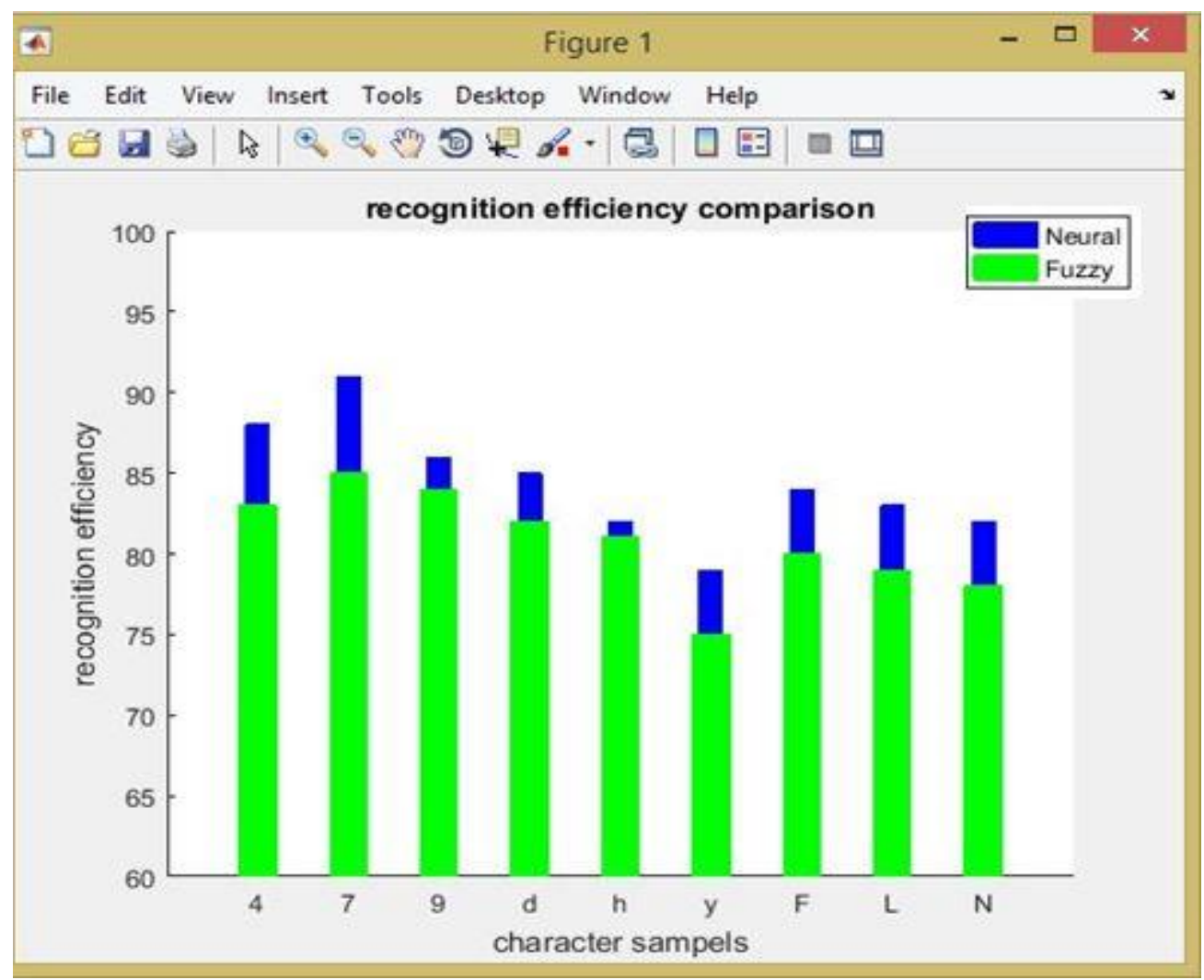

\section{CONCLUSIONS}

Figure 3. Recognition efficiency comparison

The average recognition efficiency for all characters in the $\mathrm{Ch} 74 \mathrm{~K}$ dataset using neural classification was $82 \%$ while for Fuzzy classification was $78 \%$. Figure (3) shows some character samples' with their efficiency, For Fuzzy, efficiency ranges from ( $75 \%$ for (y) character) to $(85 \%$ for digit (7)), while for neural, it ranges from (79\% for (y) character) to $(91 \%$ for digit (7)) , this because of:

5.1 Sample Selection: The Ch74K dataset contains high resolution samples of (digit (7)) versus low resolution samples for (character(y)), Training the network with these different samples for each character/ digit leads to these efficiencies. So sample selection from high resolution dataset is important to ensure the effectiveness and worth of training.

5.2 The limitation of time consuming Fuzzy classification, which leads to the size limitation to $(8 * 8$ pixel) character size and with each one of the datasets individually (digit dataset, capital character dataset and small character dataset) as dataset and each one of these datasets includes only five samples of each character, that is the reason Fuzzy classification gives less efficiency. While Neural classification was used with $(32 * 32$ pixel) character size (with 51928 training images, 22177 test images), which increases the possibility of recognition and also higher recognition efficiency than Fuzzy classification was achieved because more dataset images is taken for training.

5.3 Neural classification is more suitable for online character recognition because of it is less time consuming than Fuzzy classification. 
International Journal of Embedded Systems and Applications (IJESA), Vol 8, No.4, December 2018

5.4 The choosing of the Ch74K dataset is because it has handwritten, machine printed and natural gray and colored images which was appropriate to the work.

\section{REFERENCES}

[1] A. Yuan, G. Bai, P. Young, Y. Guo, X. Zhao, (2012) "Handwritten English word recognition based on convolution neural networks", International conference on frontiers in handwriting recognition.

[2] Pranob K Charles, V. Harsh, M. Swath, CH. Deepth, (2012) "A review on the various techniques used for optical character recognition", International journal of engineering research and application, vol.2, pp.659-662.

[3] Téofilo E. de Campos, Bodla Rakesh Babu, and Manik Varma, (2009) "Character Recognition In Natural Images”, Microsoft Research India “Scientia” 196/36 2nd Main, Sadashivnagar, Bangalore 560080 India.

[4] M. Sifuzzaman, M.R. Islam and M. Z. Ali, (2009) "Application of Wavelet Transform and its Advantages Compared to Fourier Transform”, Journal of Physical Sciences, Vol. 13, 121-134.

[5] S. Chuai-Aree, C. Lursinsap, P. Sophasathit and S. Siripant, (2001) "Fuzzy C-Mean: A Statical Feature Classification Of Text And Image Segmentation Method“, International Journal of Uncertainty, Fuzziness and Knowledge-Based Systems, Vol. 9, No. 6, 661-671.

[6] Nilay Karade, Dr. Manu Pratap Singh, and Dr. Pradeep K. Butey, (2015) "Handwritten Character Recognition Using Feed-Forward Neural Network Models“, International Journal of Computer Trends and Technology (IJCTT) - Volume 6, Issue 2, pp. 54-74.

[7] Shyla Afroge, Boshir Ahmed, and Firoz Mahmud, (2016) “ Optical Character Recognition using Back Propagation Neural Network", 2nd International Conference on Electrical, Computer \& Telecommunication Engineering (ICECTE), Rajshahi-6204, Bangladesh , 8-10.

[8] Muhammad Arif Mohamad, Dewi Nasien, Haswadi Hassan and Habibollah Haron, (2015) “ A Review on Feature Extraction and Feature Selection for Handwritten Character Recognition“, (IJACSA) International Journal of Advanced Computer Science and Applications, Vol. 6, No. 2.

[9] Mayuri Rastogi, Sarita Chaudhary, and Shiwani Agarwal, (2013) "Different Classification Techniques for Character Recognition: A Survey “, MIT International Journal of Computer Science \& Information Technology Vol. 3, No. 1, pp. 30-34, ISSN 2230-7621.

[10] Siddhi Sharma, and Neetu Singh, (2014) “Optical Character Recognition Using Artificial Neural Network Approach", International Journal of Emerging Technology and Advanced Engineering, ISSN 2250-2459, ISO 9001:2008 Certified Journal, Volume 4, Issue 11.

[11] S.V. Shelke, A. H. Dhangare and S. D. Patil, (2014) "Handwritten Character Recognition using Wavelet Transform for Feature Extraction", International Journal of Multidisciplinary Educational Research (IJMER), Vol. 3, Issue 3(7).

[12] Thamer M. Jamel and Ban M. Khammas, (2012) "Implementation Of A Sigmoid Activation Function For Neural Network Using FPGA“, Published in the 13th Scientific Conference of AlMa'moon University College, Baghdad, Iraq. 